$$
5 \cdot 7
$$

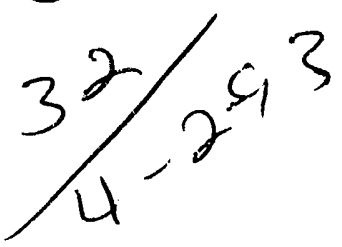

FNAL-TM--1815

DE93 010524

\title{
Effective Length Measurements of Prototype Main Injector Dipole Endpacks
}

\author{
Henry D. Glass, Bruce C. Brown and David J. Harding \\ Fermi National Accelerator Laboratory \\ P.O. Box 500, Batavia, Illinois 60510
}

\section{DISCLAIMER}

\begin{abstract}
This report was prepared as an account of work sponsored by an agency of the United States Government. Neither the United States Government nor any agency thereof, nor any of their employees, makes any warranty, express or implied, or assumes any legal liability or responsibility for the accuracy, completeness, or usefulness of any information, apparatus, product, or procuss disclosed, or represents that its use would not infringe privately owned rights. Reference herein to any specific commercial product, process, or service by trade name, trademark, manufacturer, or otherwise does not necessarily constitute or imply its endorsement, recommendation, or favoring by the United States Government or any agency thereof. The views and opinions of authors expressed herein do not necessarily state or reflect those of the United States Government or any agency thereof.
\end{abstract}

December 1992 


\title{
Effective Length Measurements of Prototype Main Injector Dipole Endpacks
}

\author{
Henry D. Glass Bruce C. Brown \\ David J. Harding \\ Fermi National Accelerator Laboratory * \\ P.O. Box 500 \\ Batavia, Mlinois 60510
}

March 3, 1993

\begin{abstract}
An endpack design has been developed for the Feimilab Main Injecior Dipole. A major part of the design process was the testing of a series of prototype removable endpacks. The magnetic parameters that were tested includeci the effective length and the field shape variation. This report presents a description of the measurement techniques and the results for the effective length. The final endpack has an effective length at $1500 \mathrm{~A}(0.29 \mathrm{~T})$ of $2.6 \pm 0.3 \mathrm{~mm}$ greater than the steel length, and the change in effective length from $1500 \mathrm{~A}$ to maximum current of $9500 \mathrm{~A}(1.74 \mathrm{~T})$ is $-1.88 \pm 0.05 \mathrm{~mm}$.
\end{abstract}

\section{Introduction}

We report measurements of the effective length for a number of endpacks which were mounted on the non-lead end of magnet IDM002, the 2nd prototype dipole for the Main Injector[1]. As of this writing, ten different endpacks have been tested. The last of these endpacks represents the final design, or something very close to it; updated versions of this report may be issued if subsequent endpacks are measured.

\footnotetext{
"Operated by the Universities Research Association under contract with the U. S. Department of Energy
} 
The endpacks were measured using the 80 inch long $(2.032 \mathrm{~m})$ Flatcoil probe. The probe has 24 turns and has a width of 0.268 inches $(6.81 \mathrm{~mm})$. The turns were spaced in a geometry which minimizes the sextupole contribution of the flux, as expanded about the probe center. What this means is that although the flux measured by each turn is proportional to the field averaged over the probe area, the total flux, summed over all turns, is very nearly proportional to the field integral along a path running down the geometrical center of the probe. The probe was mounted on a movable stand which allowed us to insert the probe up to 40 inches inside the magnet. In the baseline mode of operation, the probe records the difference in flux between zero current and current $i$ :

$$
\Delta \Phi(i)=\bar{\Phi}(i)-\bar{\Phi}(\hat{0}) .
$$

By aligning the long axis of the probe with the longitudinal $(z)$ axis of the magnet, we easily recover the integrated field cver the length of the probe:

$$
J\left(z_{1}, z_{2}\right)=\int_{z_{1}}^{z_{2}} B(z) d l=\frac{\Delta \Phi}{N w}
$$

where $N$ is the number of turns and $w$ is the probe width; $z_{1}$ ansd $z_{2}$ are the endpoint coordinates of the probe. We used a coordinate system where $z$ is zero at the first lamination of the endpack, and is positive going into the magnet. This relation between field integral and flux is correct except for a correction for the remnant field contribution. We have neglected this contribution in our analysis; future plans call for measuring the end field integral by scanning along a path on the $z$ axis with a Hall probe, which is sensitive to the remnant field.

In all of the endpack measurements we position the probe so that $z_{1}$ is far outside the magnet, in a region whe $a$ the field is negligible. One may therefore approximate $z_{1}$ as being equal to $-\infty$. The integral then becomes a function solely of the endpoint of the probe that is inside the magnet:

$$
J(z)=\int_{-\infty}^{z} B(z) d l
$$

In this case we have assumed that the probe lies along the centerline of the magnet, at $x=0$. While this is true for all measurements presented here, we vill present results in a companion report [2] where we measure the field integral as a function of $\alpha$. 


\section{Effective Length Calculation}

The total effective length for a magnet excited to a specified current $i$ is

$$
\mathcal{L}_{\text {eff }}(i)=\frac{\int_{-\infty}^{\infty} B(i, z) d l}{B_{0}(i)}
$$

where $B_{0}$ is the mean body field. The total integral may be measured using a probe which extends the entire length of the magnet, and $B_{0}$ may be measured by a probe which samples only the body field. In a high quality dipole, the body field is very uniform, only falling off as one approaches close to the ends. With this observation in mind, we can re-express Eq. 2 in terms of the steel length, $L_{0}$, and a quantity $L_{\text {eff }}$ which we call the end effective length:

$$
\mathcal{L}_{\text {eff }}(i)=L_{\bullet}+2 L_{\text {eff }}(i)
$$

The factor of 2 is present because of our definition that $L_{\text {eff }}$ is the effective length of each end of the magnet. For simplicity, when we discuss effective length in the remainder of this report, we are referring to $L_{\text {eff }}$. We devised a measurement procedure which measures $L_{\text {eff }}$ using the short 80 inch probe. If the probe is inserted a distance $z$ into the magnet, then the quantity

$$
\ell(z)=\frac{J(z)}{B_{0}}-z
$$

should become constant and approach $L_{\text {eff }}$ as $\mathrm{z}$ becomes larger. Experience with this magnet and these endpacks shows this to be true for $z$ between 10 and 20 inches. The body field, $B_{0}$, is determined by performing a linear fit to $J(z)$ :

$$
J(z)=\alpha+\beta z
$$

The slope, $\beta$, can be identified as the mean body field in the region $z_{\min }<$ $z<z_{\max }$ over which the fit is made.

The relative effective length describes the change in $L_{\text {eff }}$ with current and can be determined by choosing a reference current, $i_{0}$, and using Equation 4 to obtain

$$
\Delta \ell(i, z)=\ell(i, z)-\ell\left(i_{0}, z\right)=\frac{J(i, z)}{B_{0}(i)}-\frac{J\left(i_{0}, z\right)}{B_{0}\left(i_{0}\right)}
$$

The average of $\Delta \ell(i, z)$ for $z>z_{\min }$ is used as the value of $\Delta L_{\text {eff }}(i)$. One may see from Equation 6 that this quantity is insensitive to positioning errors in $z$, a dominant source of systematic errors. For the reference current $i_{0}$ we chose $1500 \mathrm{~A}$. 


\section{Measurement Procedure}

We chose a number of $z$-positions at which to measure $J(i, z)$, moving in 1 inch steps in $z$ for the first 4 incles of the magnet and in 2 inch steps thereafter, up to a maximum $z$ of 20 inches. At each $z$, we measured the field integral at a number of currents which included 500,1500,7000, and $9500 \mathrm{~A}$. For many measurement runs we included a number of additional currents. At each data point we recorded the measurements of the current, the $z$ position, and a quantity, $\phi$, equal to the flux divided by the current ${ }^{1}$. A VAX was used as the data acquisition computer, which ran the FLATCOIL program [6] to control the measurement process and to record data to disk files. The $z$ position was the only quantity of the measurement process under manual control, both in terms of positioning (via alignment with a steel ruler mounted on the test stand), and recording.

The probe was aligned perpendicular to the face of the laminations. It was centered at $x=0$, as measured at the endpack edge $(z=0)$. Because of the magnet curvature, this introduced an angle of $0.6^{\circ}$ between the probe axis and the magnet axis. The error due to this misalignment increases with $z$ and is dominated by the body field sextupole; at $z=20$ inches the error at $9500 \mathrm{~A}$ is

$$
\begin{aligned}
\Delta B / B & \approx b_{3} x^{2} \\
& =b_{3}(z \tan \theta)^{2} \\
& =\left(-10 \times 10^{-4}\right) \times\left(20 \times \tan \left(0.6^{\circ}\right)\right)^{2} \\
& \approx 4.4 \times 10^{-5}
\end{aligned}
$$

This is smaller than the error due to positioning of the probe in $z$. We were able to obtain, in typical runs, accuracies between 0.005 " and 0.010 ". From Equation 5, we estimate that

$$
\sigma_{J} / J \approx \sigma_{z} / z
$$

where we have used the approximation $\beta \approx J / z$. Since $\sigma_{z}$ was approximately constant in $z$, the error in $J$ is worst at low $z$; e.g. at $z=10$ we have $\sigma_{J} / J \approx 1 \times 10^{-3}$.

\footnotetext{
${ }^{1}$ We recorded $\phi$ as a first approximation method of correcting for current fluctuations. This method, although inaccurate at higher currents where the magnet saturates, does not adversely affect the measurements described here. A more desirable scheme will be employed at a future date.
} 


\section{Endpacks}

The endpacks we have measured to date are described in Table 1. The table lists their number and a brief description. References [5] should be consulted for drawings of endpack profiles.

The first two endpacks had a profile in the $(y, z)$ plane which consisted of a single cut at about $66^{\circ}$ to the horizontal. The vertex of this cut was located at about -2.5 inches in $z$. The first endpack was machined to the desired shape out of body laminations. All of the endpacks from \#2 on were made using nibbled laminations. Endpack 3 was constructed using a Borda profile [3], which sought to minimize the dependence of effective length on current. This profile was unsuccessful, as measurements presented here show, and Endpack 4 was a modification of \#3, in which an additional 1.5 " thickness of laminations was bolted on to the exterior of the endpack. An alternate strategy, employing the Rogowsky profile [4], was then used for the succeeding endpacks. Endpack 5, which was machined out of Endpack 2 , had an acceptable $L_{\text {eff }}$ current dependence, but its absolute effective length was smaller than desired. Some outer laminations were removed from this endpack, and this became Endpack 6, which had an acceptable $L_{\text {eff }}$ behavior.

Endpacks beyond \#6 were modifications designed to address a secondary requirement, which was to adjust the endpack sextupole to an acceptable value. This topic is addressed in another report [2]. Fortuitously, the efforts at minimizing the sextupole also had the advantage of further reducing the effective length variation.

\section{Field Profiles}

As a first step in understanding the behavior of the end field, we can calculate the field as a function of $z$ from measurements of $J(z)$. This is simply done by approximating the derivaiive of $J$ :

$$
\overline{B(z)}=\frac{d J}{d z} \approx \frac{j\left(z_{1}\right)-J\left(z_{2}\right)}{z_{1}-z_{2}},
$$

where $\mathrm{z}$ is midway between the points $z_{1}$ and $z_{2}$ at which we have positioned the probe. A uscful quantity to examine is the field profile normalized to its value deep inside the body. In Figure 1 we show $B_{n}=B / B_{b o d y}$ vs $z$ for each of 4 currents for Endpack 9. The region where the profiles for 


\begin{tabular}{|l|l|}
\hline number & description \\
\hline 1 & Machined endpack \\
2 & Nibbled; same profile as 1 \\
3 & Borda profile \\
4 & same as 3, with additional 1.5" laminations \\
5 & approximate Rogowsky profile \\
6 & same as 5 but with 9 outer lams removed \\
7 & same as 6 but with noses reduced in size \\
8 & same as 7 with noses further reduced \\
9 & same as 8 with noses gone except for small shims \\
10 & same as 9 with gap between shims widened \\
\hline
\end{tabular}

Table 1: Endpack descriptions

low and high current are most different occurs where the field begins to drop off, around 2 to 4 inches. This can be seen more readily by plotting $B_{\text {rel }}(i, z)=B_{n}(i, z) / B_{n}\left(i_{0}, z\right)$, where we choose $i_{0}$ to be some suitable low current. In our analysis we chose $i_{0}=1500 \mathrm{~A}$ because its profile was similar to $500 \mathrm{~A}$ but had higher signal to noise.

In Figure 2 we plot $B_{\text {rel }}$ vs $z$ for 7000 and $9500 \mathrm{~A}$. The minimum in the data at $z=1.5$ inches presumably corresponds to the point where the relative degree of saturation at high currents is strongest.

Figure 3 shows $B_{\text {rel }}(9500 \mathrm{~A})$ vs $z$ for some representative endpacks. We see that Endpack 9 exhibits the least amount of saturation at the end. As might be expected, the endpacks having a shallow minimum near $z=2^{\prime \prime}$ also have a small change of $L_{\text {eff }}$ with current.

The profiles are also a useful way of estimating a reasonable value for $z_{\min }$, the lower bound in $z$ for determining a fit to the body field via Equation 5 . We have used $z_{\min }=12^{\prime \prime}$ in our analysis; as justification, we note that, within measurement error, $B_{n} \approx 1$ for $z>12^{\prime \prime}$ for all endpacks.

\section{Effective Length Measurements}

Using the procedure described in Section 2 we calculated $L_{\text {eff }}(i)$ and $\Delta L_{\text {eff }}(i)$ for each endpack. The results are listed in Tables 3 through 13. Each table corresponds to a measurement sequence on a given endpack. Some end- 


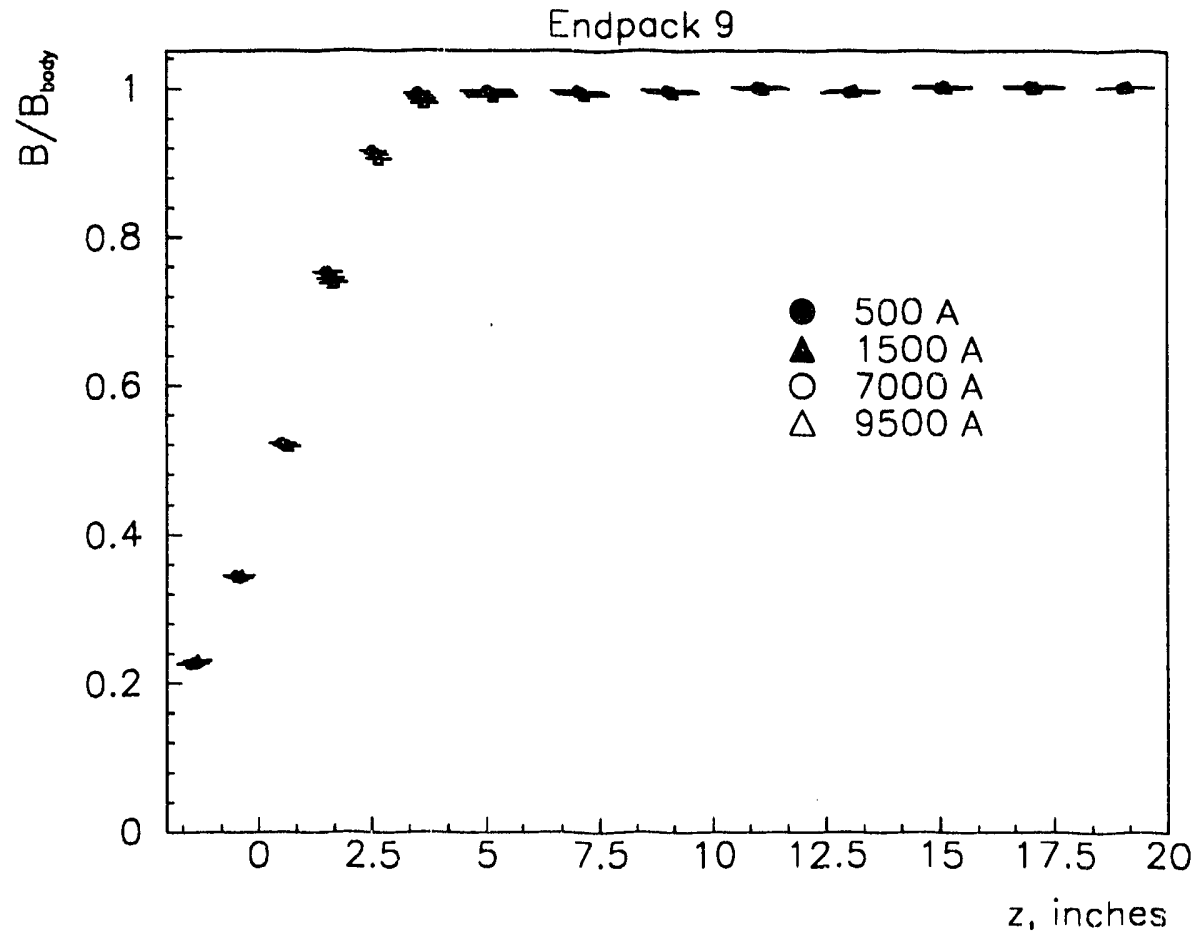

Figure 1: The field profiles, $B / B_{b o d y}$ vs $z$ for Endpack 9 at several different currents.

packs were measured more than once, usually to determine measurement repeatability or to understand the effect of varying some of the measurement conditions. Each row in the tables lists the mean measured current and standard deviation; $B_{0}$, the fitted value of body field divided by the mean current, in units of gauss/ampere; the error in the fit; the value of $L_{\text {eff }}$ in $\mathrm{mm}$ and its error estimate; and the value of $\Delta L_{\text {eff }}$ (relative to 1500 A) in $\mathrm{mm}$, and its standard deviation.

Table 2 lists for each endpack measurement a number of ancillary parameters, which include $z_{\min }, z_{\max }$, the summary file number, and $\sigma_{z}$ (inches). We always used the maximum measured value of $z$ for $z_{\max }$, which was usually $20^{\prime \prime}$, except for some early measurements ${ }^{2}$ in which we measured the flux out to $40^{\prime \prime}$. The file number references files FCMnnn.002 in direc-

\footnotetext{
${ }^{2}$ Endpack 1, file 144; Endpack 2, file 568; and Endpack 4, file 1514
} 


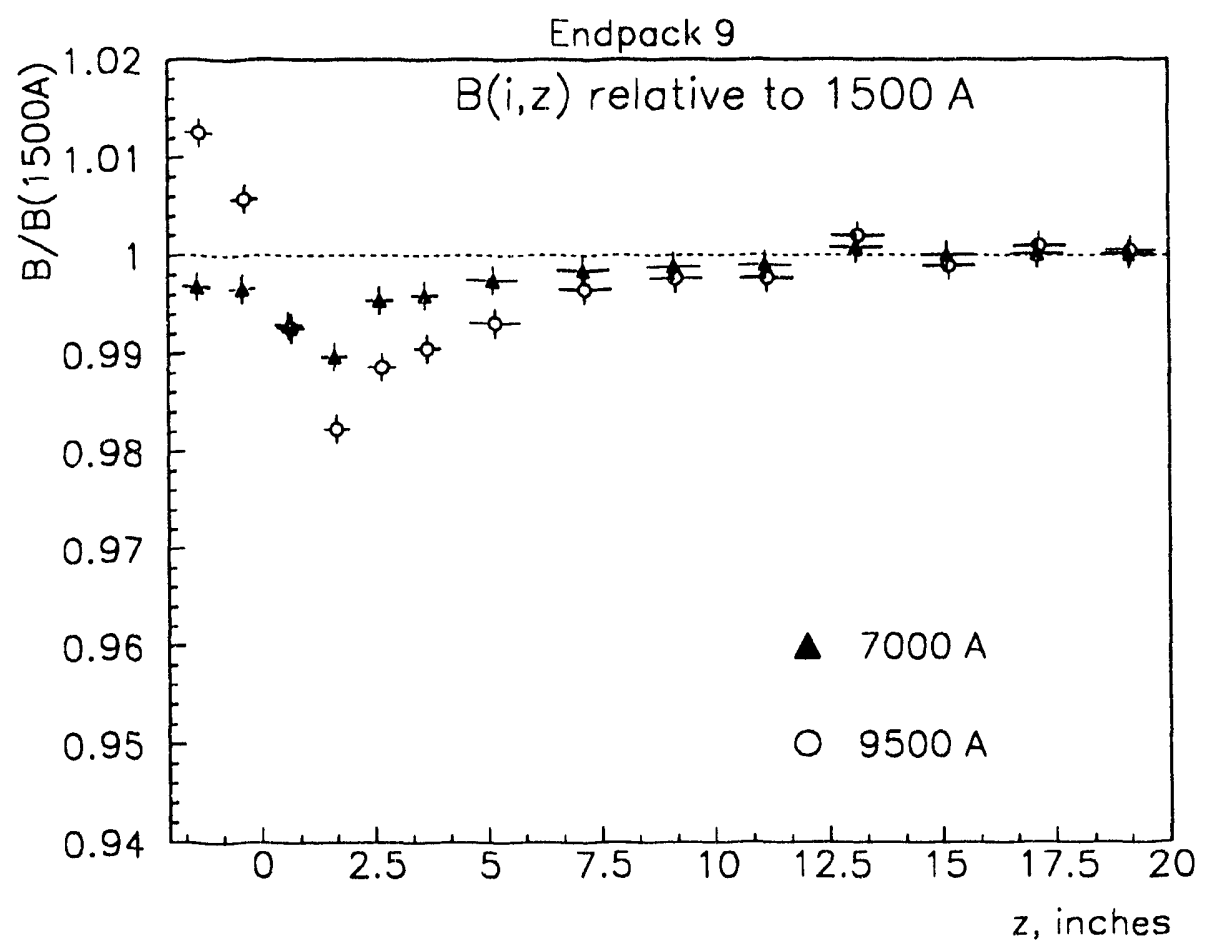

Figure 2: The relative field profiles $B_{\text {rel }}$ vs $z$ for Endpack 9 at 7000 and $9500 \mathrm{~A}$.

tory MDTF08::MDTF08\$ROOT:[GLASS.FLATCOIL], which contain summaries of the original flux data. An additional parameter used in the analysis is an estimate of the relative flux error, $\sigma_{J} / J$; we used a value of $1 \times 10^{-4}$ throughout our final analysis. This turns out in most cases to be an overestimate (see Section 7) but does not significantly affect the analysis.

Plots of $L_{\text {eff }}$ vs current are shown in Figures 4 and 5. A value of zero for $L_{\text {eff }}$ means that the effective length is equal to the physical length of the steel; a positive value of $L_{\text {eff }}$ means that the effective length is longer than the physical length. Endpacks 9 and 10 had results that were sufficiently similar that only \#9 was plotted.

A test of the long-term repeatability of these measurements was done by comparing measurements of Endpack 1 taken one year apart. During this interval, not only had the endpack been removed and subsequently 


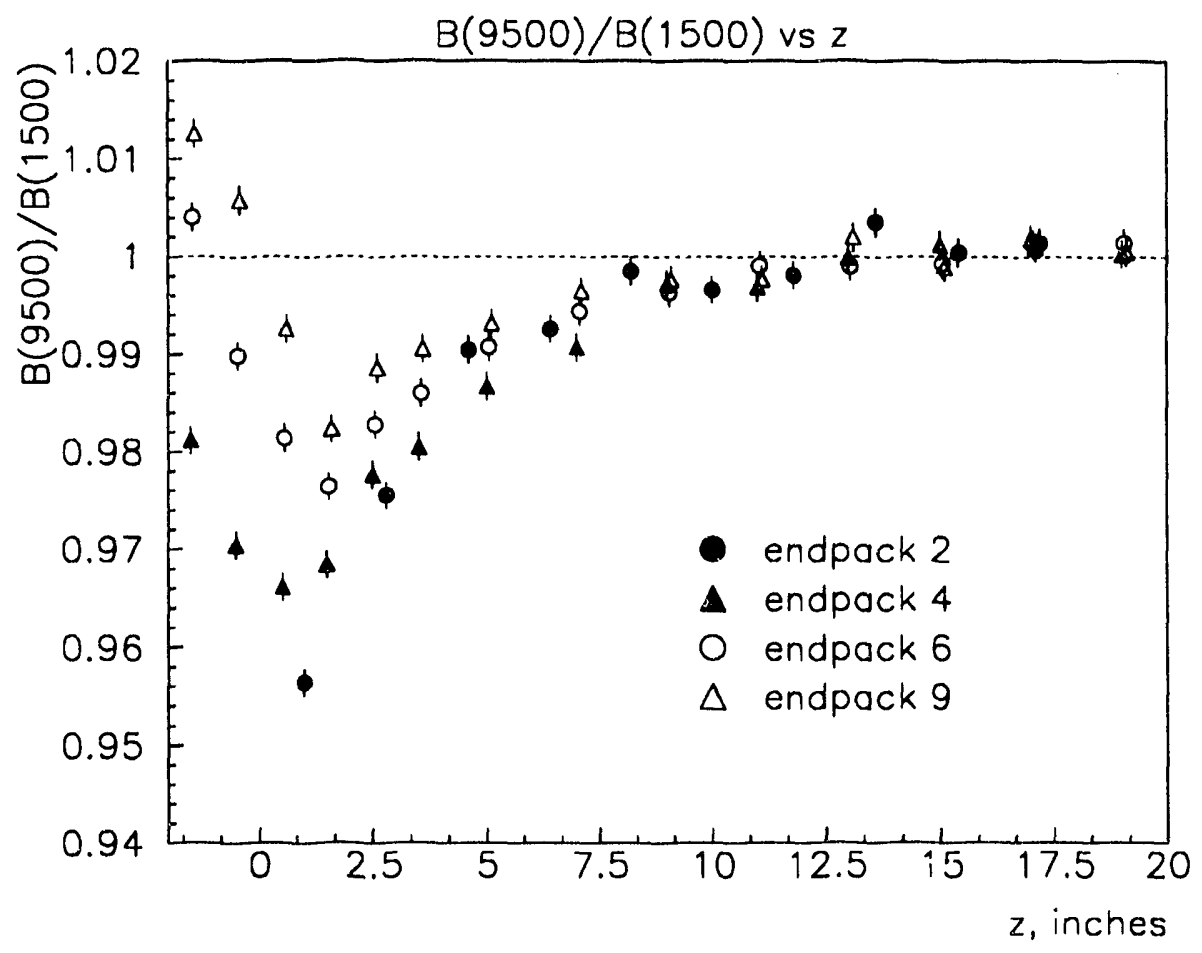

Figure 3: The relative field profile at $9500 \mathrm{~A}$ for selected endpacks.

remounted, but the magnet itself had been removed from the test stand for a period of time. The results are shown in Figure 6 . The agreement between the two data sets is very good, except at the lowest currents, where some data acquisition problems were encountered in the earlier data.

\section{Error estimates}

A set of measurements was taken in order to understand the contribution of $z$ positioning errors to the total error in the flux. First, we took four runs at $1500 \mathrm{~A}$ with the probe fixed at $z=20^{\prime \prime}$. These runs produced a standard deviation of the flux equal to $\sigma_{\Phi} / \Phi=1.6 \times 10^{-5}$. This error was identified as being due to a combination of electronic readout noise and accuracy in magnet current readout. Another four runs were then taken, in which the probe was removed and then repositioned at $z=20^{\prime \prime}$ prior to each run. In 


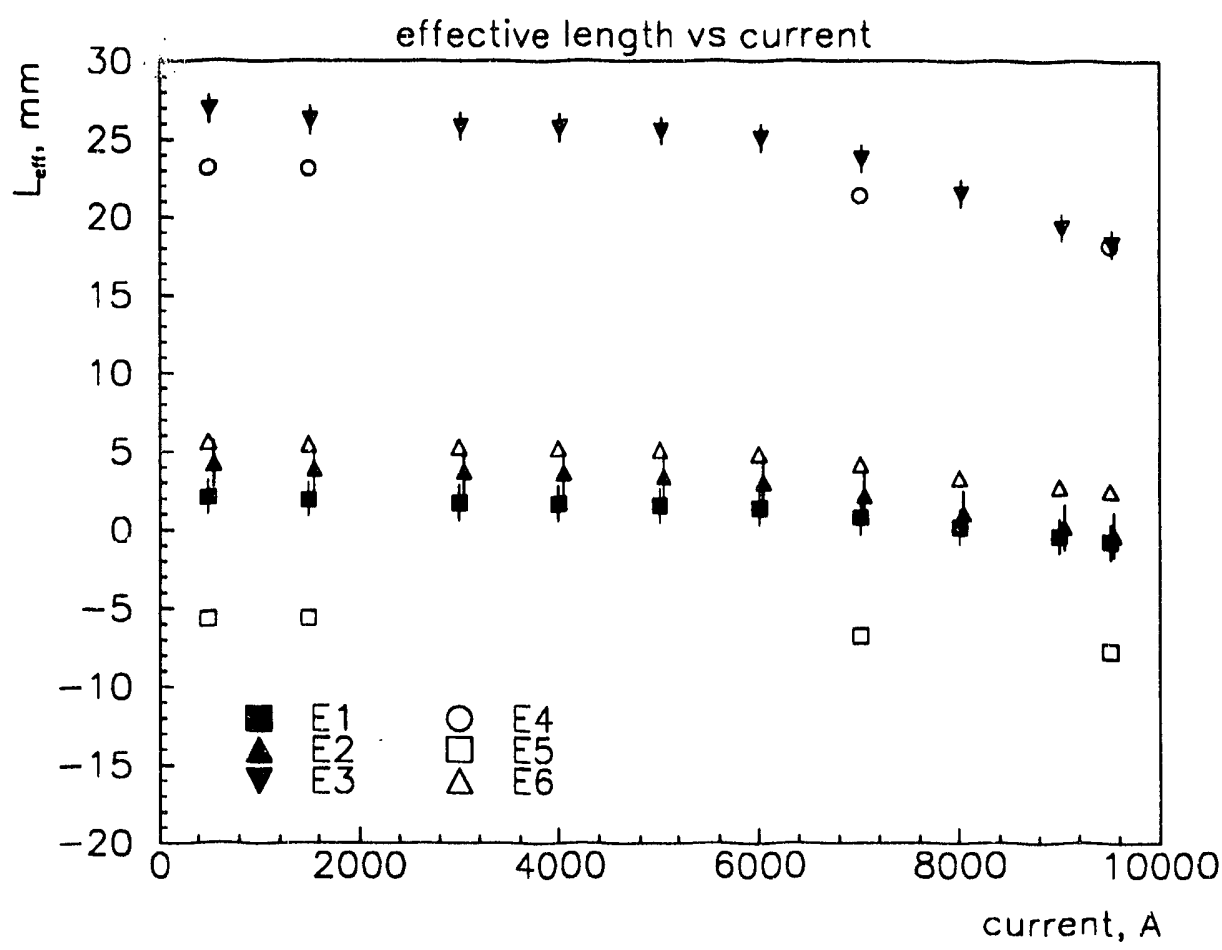

Figure 4: The effective length as a function of magnet current for Endpacks 1-6.

this case we obtained $\sigma_{\Phi} / \Phi=3.6 \times 10^{-4}$, which we attribute to the combined influence of noise plus $z$ positioning errors. The error due to $z$ positioning alone is estimated from

$$
\sigma_{\Phi}^{2}=c_{\text {noise }}^{2}+\sigma^{2}(z)
$$

This leads to an error in $z$ positioning of $0.007^{\prime \prime}$. We also repeated these measurements at $z=10^{\prime \prime}$ and obtained $\sigma_{z}=0.003^{\prime \prime}$. Averaging these two results gives $0.005^{\prime \prime}(0.13 \mathrm{~mm})$, which leads to a systematic error in $L_{\text {eff }}$ of $0.37 \mathrm{~mm}$ (see Eq. 7, below).

The error in the current that we report is the standard deviation for all of the current readings at a particular nominal current setting. This value is typically less than $0.5 \mathrm{~A}$, and is mainly due to the stability of the power supply controller module, power supply noise, and current readcut noise. The error in $B_{0} / i$ is the least squares fitting error in the fit of flux vs $z$. 


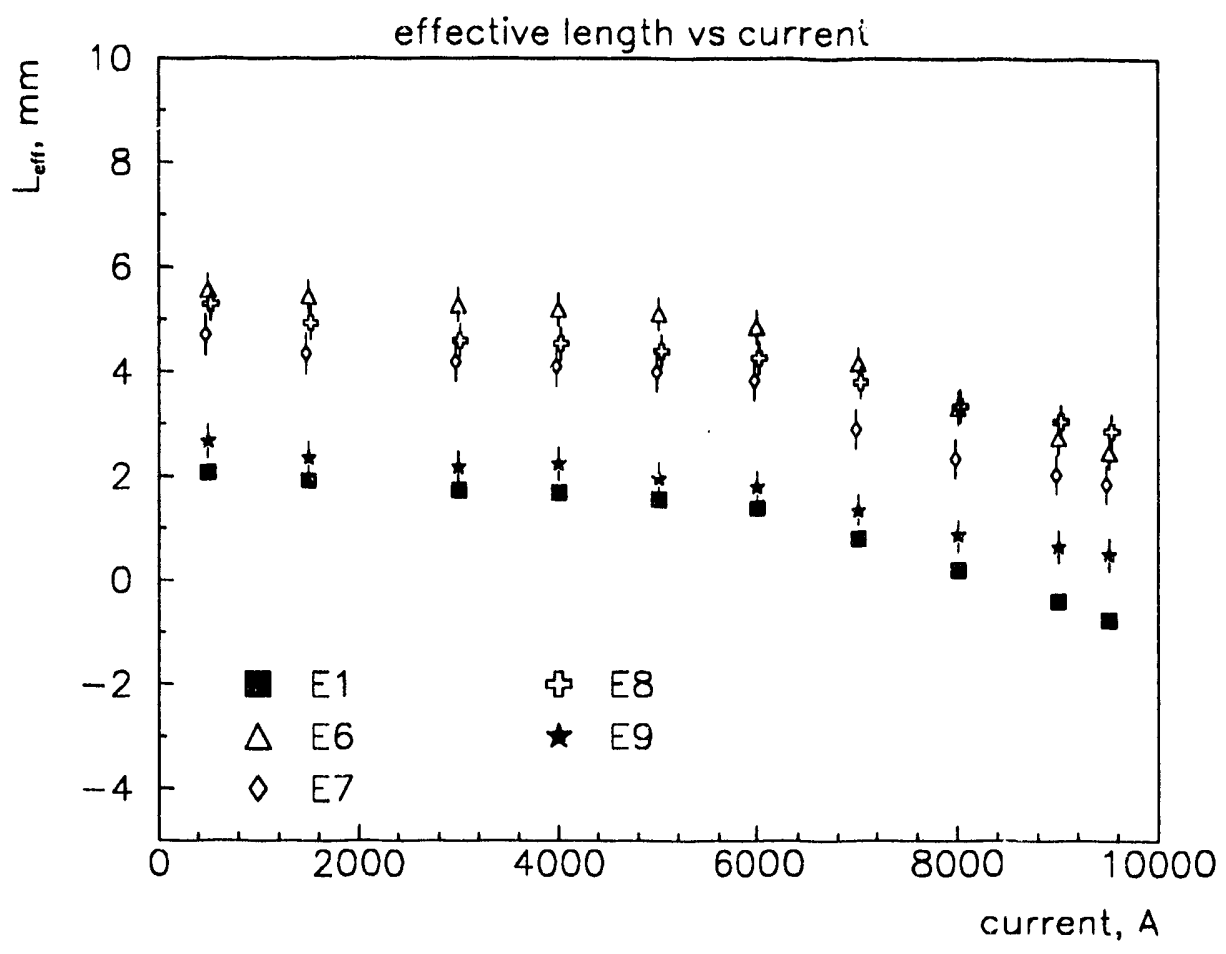

Figure 5: The effective length vs current for Endpacks 6-9, plus Endpack 1 for comparison.

The error reported for $L_{\text {eff }}$ is the combined statistical and systematic error. Equation 4 is applied at each data point between $z_{\min }$ and $z_{\max }$, and the standard deviation in $L_{\text {eff }}$ is used as the statistical error. The systematic error is calculated from

$$
\sigma_{L}^{2}(s y s)=\left[\left(\frac{\sigma_{J}}{J}\right)^{2}+\left(\frac{\sigma_{B_{0}}}{B_{0}}\right)^{2}\right] \overline{z^{2}}+\sigma_{z}^{2}
$$

The error in $z$ not only appears explicitly above as the term $\sigma_{z}$ but also implicitly, since $\sigma_{B_{0}}$ depends strongly on it. In contrast, the errors reported for $\Delta L_{\text {eff }}$ are only statistical, as the systematic errors are essentially the same between currents.

Plots of the current dependence of $\Delta L_{\text {eff }}$ are shown in Figures 7 and 8. The overall change in $L_{\text {eff }}$ over the entire range of currents decreases 


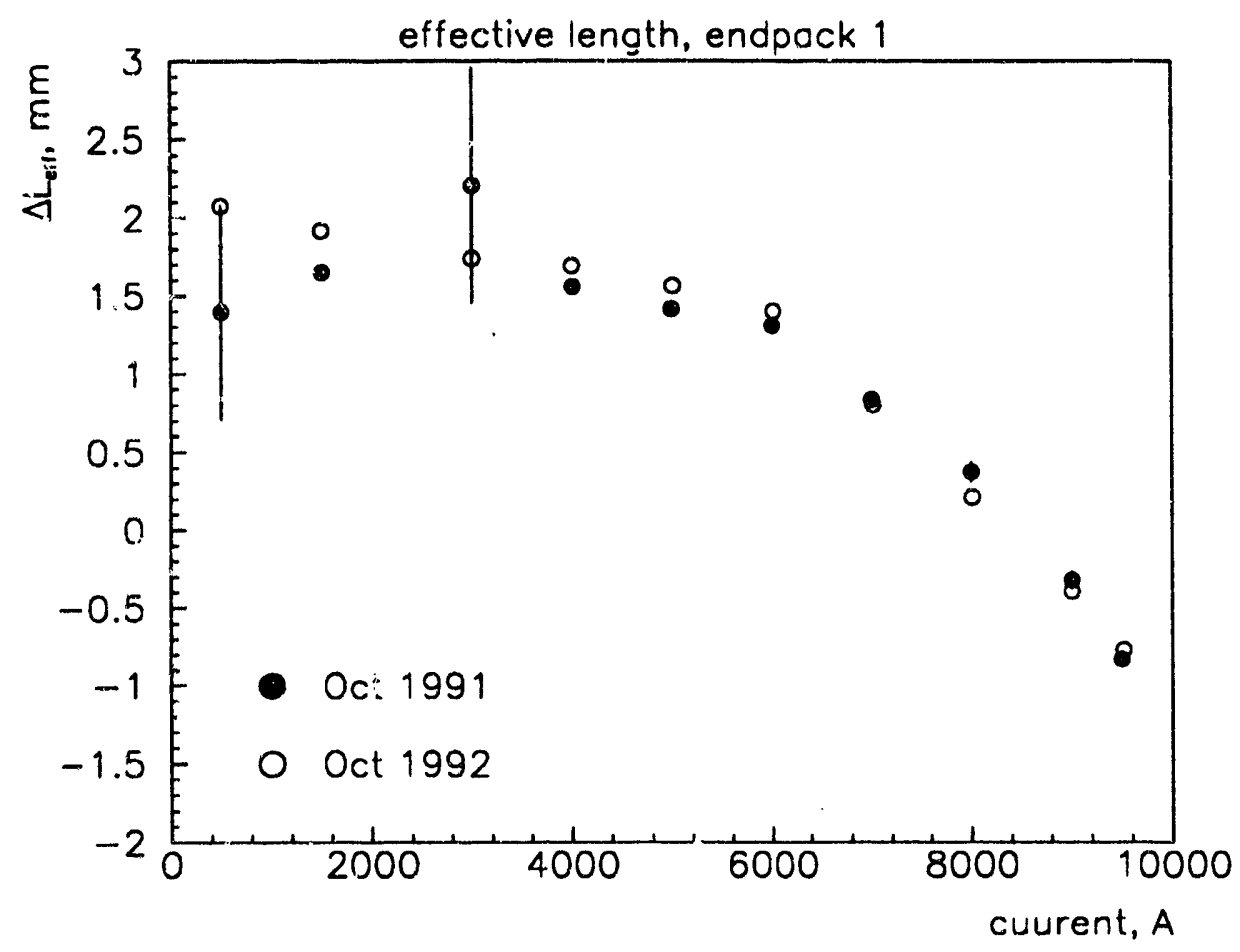

Figure 6: A comparison of the effective length measurements taken on Endpack 1 at two different times.

steadily from Endpacks 6-9, which was the intended effect. (Endpack 10 was sufficiently close to 9 that it was left out of the plot.) The final endpacks meet $\Delta L_{\text {eff }}$ requirements. 


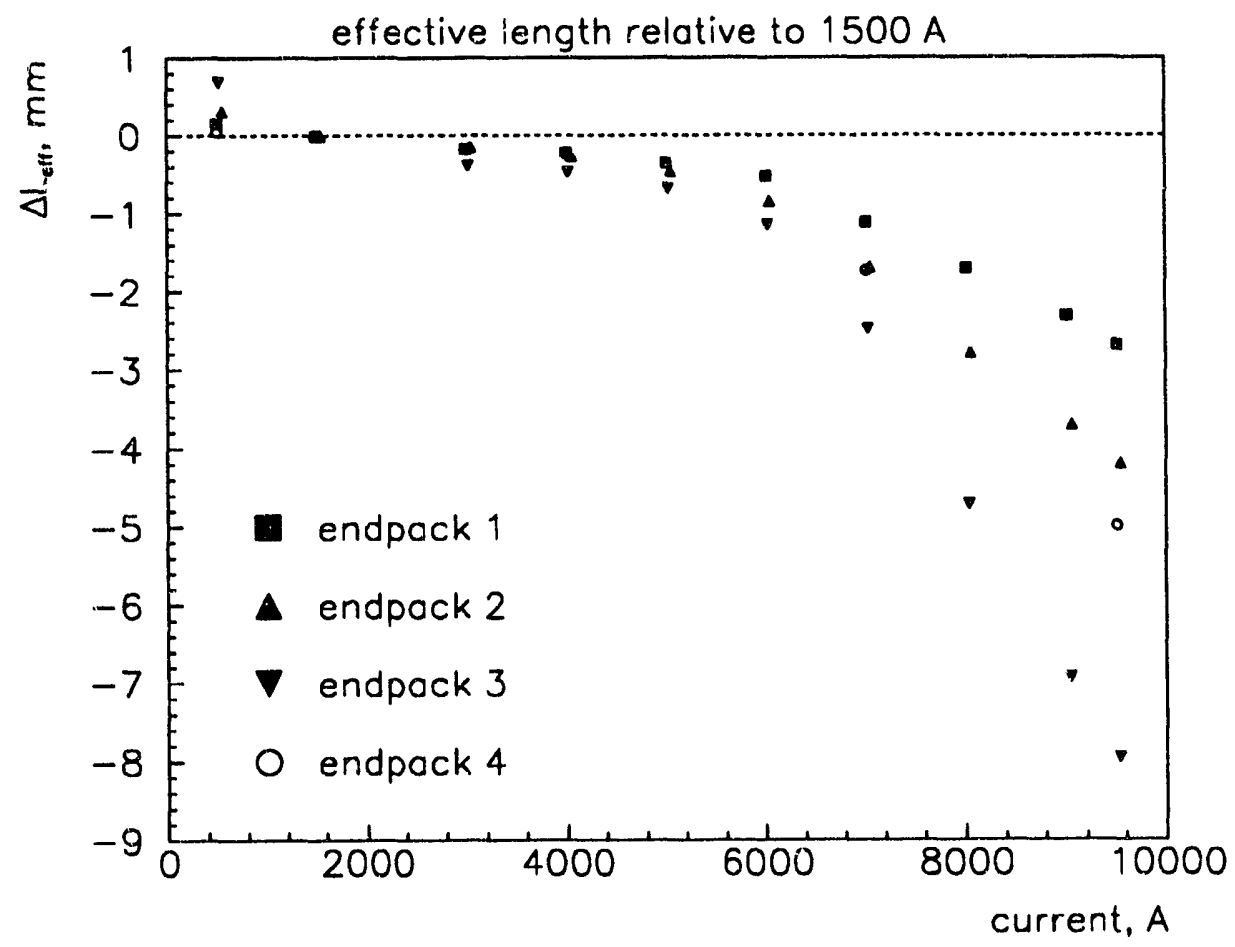

Figure 7: The effective length relative to $1500 \mathrm{~A}$ for Endpacks 1-4. 


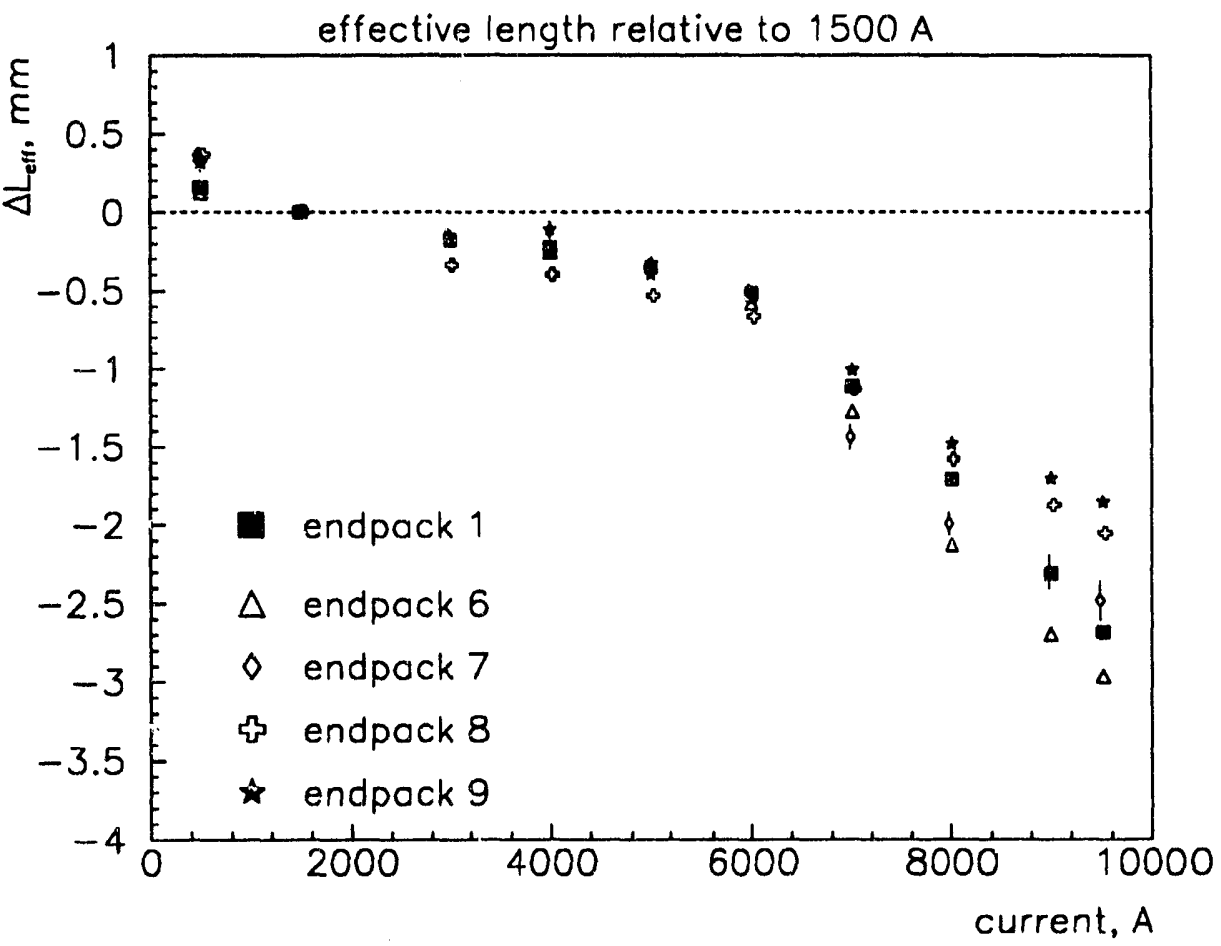

Figure 8: The effective length relative to $1500 \mathrm{~A}$ for Endpack 1 and 6-9. The final endpack has the most desirable $\Delta L_{\text {eff }}$ behavior. 


\begin{tabular}{|c|c|c|c|c|}
\hline Endpack & file & $z_{\min }$ & $z_{\max }$ & $\sigma_{z}$ \\
\hline 1 & 144 & 15.0 & 39.6 & 0.010 \\
1 & 2074 & 12.0 & 20.0 & 0.015 \\
2 & 568 & 15.0 & 40.1 & 0.030 \\
3 & 1270 & 25.0 & 40.0 & 0.010 \\
4 & 1391 & 12.0 & 20.0 & 0.006 \\
5 & 1530 & 12.0 & 20.0 & 0.004 \\
6 & 1818 & 12.0 & 20.0 & 0.004 \\
7 & 1914 & 12.0 & 20.0 & 0.005 \\
8 & 2147 & 12.0 & 20.0 & 0.004 \\
9 & 2253 & 12.0 & 20.0 & 0.004 \\
10 & 2380 & 12.0 & 20.0 & 0.004 \\
\hline
\end{tabular}

Table 2: Auxiliary parameters for endpack data: summary file number, region in $z$ in which fit for $B_{0}$ is performed, and estimated $z$ position error.

\begin{tabular}{|r|r|r|r|}
\hline current, $\mathrm{A}$ & $B_{0} / i$, gauss $/ \mathrm{A}$ & $L_{\text {eff }}, \mathrm{mm}$ & $\Delta L_{\text {eff }}, \mathrm{mm}$ \\
\hline $499.51 \pm 0.19$ & $1.93086 \pm 0.00100$ & $1.397 \pm 0.905$ & $-0.254 \pm 0.686$ \\
$1503.59 \pm 0.04$ & $1.94247 \pm 0.00101$ & $1.651 \pm 0.523$ & $0.000 \pm 0.000$ \\
$2998.89 \pm 0.11$ & $1.94234 \pm 0.00101$ & $2.207 \pm 0.682$ & $0.556 \pm 0.751$ \\
$4003.17 \pm 0.22$ & $1.94439 \pm 0.00101$ & $1.557 \pm 0.536$ & $-0.094 \pm 0.047$ \\
$4992.89 \pm 0.11$ & $1.94349 \pm 0.00101$ & $1.414 \pm 0.526$ & $-0.236 \pm 0.039$ \\
$5998.90 \pm 0.20$ & $1.94118 \pm 0.00101$ & $1.307 \pm 0.523$ & $-0.344 \pm 0.034$ \\
$6998.12 \pm 0.14$ & $1.93480 \pm 0.00100$ & $0.834 \pm 0.517$ & $-0.816 \pm 0.032$ \\
$8000.66 \pm 0.29$ & $1.90808 \pm 0.00099$ & $0.373 \pm 0.516$ & $-1.278 \pm 0.066$ \\
$9006.11 \pm 0.20$ & $1.86310 \pm 0.00096$ & $-0.322 \pm 0.523$ & $-1.972 \pm 0.053$ \\
$9498.39 \pm 0.16$ & $1.83639 \pm 0.00095$ & $-0.828 \pm 0.506$ & $-2.478 \pm 0.050$ \\
\hline
\end{tabular}

Table 3: Effective length results for Endpack 1 (data file 144) 


\begin{tabular}{|r|r|r|r|}
\hline current, $\mathrm{A}$ & $B_{0} / i$, gauss $/ \mathrm{A}$ & $L_{\text {eff }}, \mathrm{mm}$ & $\Delta L_{\text {eff }}, \mathrm{mm}$ \\
\hline $497.42 \pm 0.18$ & $1.93788 \pm 0.00465$ & $2.075 \pm 1.118$ & $0.158 \pm 0.018$ \\
$1495.69 \pm 0.14$ & $1.94948 \pm 0.00468$ & $1.916 \pm 1.122$ & $0.000 \pm 0.000$ \\
$2998.75 \pm 0.12$ & $1.95220 \pm 0.00468$ & $1.739 \pm 1.124$ & $-0.178 \pm 0.015$ \\
$4002.08 \pm 0.25$ & $1.95153 \pm 0.00468$ & $1.692 \pm 1.123$ & $-0.224 \pm 0.013$ \\
$5006.33 \pm 0.36$ & $1.95043 \pm 0.00468$ & $1.563 \pm 1.122$ & $-0.353 \pm 0.012$ \\
$6008.79 \pm 0.52$ & $1.94795 \pm 0.00467$ & $1.397 \pm 1.120$ & $-0.519 \pm 0.010$ \\
$7011.95 \pm 0.28$ & $1.94142 \pm 0.00464$ & $0.801 \pm 1.120$ & $-1.116 \pm 0.011$ \\
$8010.70 \pm 0.44$ & $1.91421 \pm 0.00457$ & $0.209 \pm 1.122$ & $-1.707 \pm 0.038$ \\
$9009.39 \pm 0.56$ & $1.86867 \pm 0.00446$ & $-0.393 \pm 1.123$ & $-2.310 \pm 0.040$ \\
$9513.94 \pm 0.34$ & $1.84056 \pm 0.00439$ & $-0.768 \pm 1.124$ & $-2.684 \pm 0.035$ \\
\hline
\end{tabular}

Table 4: Effective length results for Endpack 1 (data file 2074)

\begin{tabular}{|r|r|r|r|}
\hline current, $\mathrm{A}$ & $B_{0} / i$, gauss $/ \mathrm{A}$ & $L_{\text {eff }}, \mathrm{mm}$ & $\Delta L_{\text {eff }}, \mathrm{mm}$ \\
\hline $499.61 \pm 0.20$ & $1.92328 \pm 0.00279$ & $4.182 \pm 1.480$ & $0.308 \pm 0.060$ \\
$1503.70 \pm 0.15$ & $1.93484 \pm 0.00280$ & $3.874 \pm 1.466$ & $0.000 \pm 0.000$ \\
$2999.14 \pm 0.20$ & $1.93727 \pm 0.00280$ & $3.724 \pm 1.461$ & $-0.150 \pm 0.017$ \\
$4003.52 \pm 0.30$ & $1.93678 \pm 0.00280$ & $3.607 \pm 1.463$ & $-0.266 \pm 0.026$ \\
$4993.46 \pm 0.25$ & $1.93564 \pm 0.00280$ & $3.416 \pm 1.461$ & $-0.458 \pm 0.033$ \\
$5999.27 \pm 0.38$ & $1.93338 \pm 0.00279$ & $3.031 \pm 1.474$ & $-0.843 \pm 0.048$ \\
$6998.69 \pm 0.43$ & $1.92669 \pm 0.00278$ & $2.179 \pm 1.459$ & $-1.695 \pm 0.042$ \\
$8001.31 \pm 0.40$ & $1.90054 \pm 0.00274$ & $1.085 \pm 1.452$ & $-2.789 \pm 0.039$ \\
$9006.85 \pm 0.46$ & $1.85558 \pm 0.00267$ & $0.174 \pm 1.454$ & $-3.700 \pm 0.044$ \\
$9499.32 \pm 0.58$ & $1.82866 \pm 0.00263$ & $-0.320 \pm 1.453$ & $-4.193 \pm 0.054$ \\
\hline
\end{tabular}

Table 5: Effective length results for Endpack 2 


\begin{tabular}{|r|r|r|r|}
\hline current, $\mathrm{A}$ & $B_{0} / i$, gauss $/ \mathrm{A}$ & $L_{\text {eff }}, \mathrm{mm}$ & $\Delta \overline{L_{\text {eff }}, \mathrm{mm}}$ \\
\hline $511.63 \pm 0.42$ & $1.92883 \pm 0.00188$ & $\mathbf{2 6 . 9 1 9} \pm 0.879$ & $0.686 \pm 0.014$ \\
$1.514 .79 \pm 0.37$ & $1.94086 \pm 0.00189$ & $26.233 \pm 0.881$ & $0.000 \pm 0.000$ \\
$3017.47 \pm 0.47$ & $1.94363 \pm 0.00189$ & $25.844 \pm 0.879$ & $-0.389 \pm 0.009$ \\
$4020.53 \pm 0.51$ & $1.94305 \pm 0.00189$ & $25.761 \pm 0.875$ & $-0.473 \pm 0.028$ \\
$5024.66 \pm 0.49$ & $1.94195 \pm 0.00189$ & $25.550 \pm 0.875$ & $-0.683 \pm 0.026$ \\
$6031.73 \pm 0.23$ & $1.93970 \pm 0.00189$ & $25.086 \pm 0.874$ & $-1.148 \pm 0.028$ \\
$7030.32 \pm 0.35$ & $1.93252 \pm 0.00188$ & $23.744 \pm 0.877$ & $-2.490 \pm 0.014$ \\
$8033.40 \pm 0.34$ & $1.90479 \pm 0.00184$ & $21.518 \pm 0.879$ & $-4.715 \pm 0.023$ \\
$9036.97 \pm 0.43$ & $1.85845 \pm 0.00179$ & $19.300 \pm 0.881$ & $-6.934 \pm 0.047$ \\
$9537.16 \pm 0.66$ & $1.83022 \pm 0.00177$ & $18.280 \pm 0.883$ & $-7.953 \pm 0.050$ \\
\hline
\end{tabular}

Table 6: Effective length results for Endpack 3

\begin{tabular}{|r|r|r|r|}
\hline current, A & $B_{0} / i$, gauss $/ \mathrm{A}$ & $L_{\text {eff }}, \mathrm{mm}$ & $\Delta L_{\text {eff }}, \mathrm{mm}$ \\
\hline $497.15 \pm 0.22$ & $1.93361 \pm 0.00202$ & $23.166 \pm 0.488$ & $0.048 \pm 0.025$ \\
$1495.13 \pm 0.08$ & $1.94511 \pm 0.00203$ & $23.118 \pm 0.480$ & $0.000 \pm 0.000$ \\
$7010.92 \pm 0.32$ & $1.93649 \pm 0.00201$ & $21.388 \pm 0.471$ & $-1.730 \pm 0.050$ \\
$9512.26 \pm 0.39$ & $1.83522 \pm 0.00189$ & $18.129 \pm 0.471$ & $-4.989 \pm 0.024$ \\
\hline
\end{tabular}

Table 7: Effective length results for Endpack 4

\begin{tabular}{|r|r|r|r|}
\hline current, $\mathrm{A}$ & $B_{0} / i$, gauss $/ \mathrm{A}$ & $L_{\text {eff }}, \mathrm{mm}$ & $\Delta L_{\text {eff }}, \mathrm{mm}$ \\
\hline $497.19 \pm 0.12$ & $1.93506 \pm 0.00130$ & $-5.683 \pm 0.299$ & $-0.058 \pm 0.093$ \\
$1495.24 \pm 0.18$ & $1.94475 \pm 0.00131$ & $-5.625 \pm 0.314$ & $0.000 \pm 0.000$ \\
$7010.78 \pm 0.48$ & $1.93769 \pm 0.00130$ & $-6.741 \pm 0.299$ & $-1.116 \pm 0.086$ \\
$9512.89 \pm 0.44$ & $1.83726 \pm 0.00123$ & $-7.749 \pm 0.300$ & $-2.124 \pm 0.084$ \\
\hline
\end{tabular}

Table 8: Effective length results for Endpack 5 


\begin{tabular}{|r|r|r|r|}
\hline current, $\mathrm{A}$ & $B_{0} / i$, gauss $/ \mathrm{A}$ & $L_{\epsilon f f}, \mathrm{~mm}$ & $\Delta L_{\text {eff }}, \mathrm{mm}$ \\
\hline $497.16 \pm 0.08$ & $1.93653 \pm 0.00134$ & $5.564 \pm 0.315$ & $0.124 \pm 0.023$ \\
$1495.45 \pm 0.10$ & $1.94709 \pm 0.00135$ & $5.441 \pm 0.319$ & $0.000 \pm 0.000$ \\
$2998.24 \pm 0.18$ & $1.94943 \pm 0.00135$ & $5.275 \pm 0.319$ & $-0.166 \pm 0.010$ \\
$4001.51 \pm 0.39$ & $1.94889 \pm 0.00135$ & $5.186 \pm 0.319$ & $-0.255 \pm 0.011$ \\
$5005.58 \pm 0.32$ & $1.94744 \pm 0.00135$ & $5.107 \pm 0.316$ & $-0.333 \pm 0.021$ \\
$6008.26 \pm 0.32$ & $1.94518 \pm 0.00135$ & $4.858 \pm 0.317$ & $-0.582 \pm 0.010$ \\
$7011.02 \pm 0.32$ & $1.93848 \pm 0.00134$ & $4.164 \pm 0.317$ & $-1.276 \pm 0.018$ \\
$8010.05 \pm 0.19$ & $1.91133 \pm 0.00132$ & $3.312 \pm 0.319$ & $-2.129 \pm 0.028$ \\
$9008.54 \pm 0.43$ & $1.86459 \pm 0.00128$ & $2.743 \pm 0.315$ & $-2.698 \pm 0.044$ \\
$9513.56 \pm 0.40$ & $1.83580 \pm 0.00126$ & $2.474 \pm 0.314$ & $-2.967 \pm 0.038$ \\
\hline
\end{tabular}

Table 9: Effective length results for Endpack 6

\begin{tabular}{|r|r|r|r|}
\hline current, $\mathrm{A}$ & $B_{0} / i$, gauss $/ \mathrm{A}$ & $L_{\text {eff }}, \mathrm{mm}$ & $\Delta L_{\text {eff }}, \mathrm{mm}$ \\
\hline $497.58 \pm 0.24$ & $1.93518 \pm 0.00163$ & $4.708 \pm 0.393$ & $0.364 \pm 0.031$ \\
$1495.84 \pm 0.14$ & $1.94679 \pm 0.00164$ & $4.344 \pm 0.389$ & $0.000 \pm 0.000$ \\
$2998.78 \pm 0.10$ & $1.94907 \pm 0.00164$ & $4.193 \pm 0.388$ & $-0.152 \pm 0.015$ \\
$4002.25 \pm 0.21$ & $1.94857 \pm 0.00164$ & $4.104 \pm 0.385$ & $-0.241 \pm 0.021$ \\
$5006.41 \pm 0.18$ & $1.94734 \pm 0.00164$ & $3.996 \pm 0.387$ & $-0.348 \pm 0.013$ \\
$6008.93 \pm 0.39$ & $1.94495 \pm 0.00163$ & $3.838 \pm 0.386$ & $-0.507 \pm 0.015$ \\
$7012.08 \pm 0.17$ & $1.94025 \pm 0.00163$ & $2.907 \pm 0.373$ & $-1.438 \pm 0.078$ \\
$8010.80 \pm 0.20$ & $1.91304 \pm 0.00160$ & $2.350 \pm 0.373$ & $-1.994 \pm 0.073$ \\
$9009.56 \pm 0.36$ & $1.86607 \pm 0.00156$ & $2.0 .16 \pm 0.371$ & $-2.298 \pm 0.112$ \\
$9514.37 \pm 0.17$ & $1.83738 \pm 0.00154$ & $1.862 \pm 0.372$ & $-2.482 \pm 0.127$ \\
\hline
\end{tabular}

Table 10: Effective length results for Endpack 7 


\begin{tabular}{|r|r|r|r|}
\hline current, $\mathrm{A}$ & $B_{0} / i$, gauss $/ \mathrm{A}$ & $L_{\text {ef } f}, \mathrm{~mm}$ & $\Delta L_{\text {eff }}, \mathrm{mm}$ \\
\hline $497.30 \pm 0.31$ & $1.93358 \pm 0.00134$ & $5.301 \pm 0.313$ & $0.367 \pm 0.031$ \\
$1495.48 \pm 0.21$ & $1.94506 \pm 0.00135$ & $4.934 \pm 0.312$ & $0.000 \pm 0.000$ \\
$2998.38 \pm 0.19$ & $1.94825 \pm 0.00135$ & $4.596 \pm 0.311$ & $-0.338 \pm 0.004$ \\
$4001.88 \pm 0.23$ & $1.94762 \pm 0.00135$ & $4.535 \pm 0.309$ & $-0.399 \pm 0.014$ \\
$5005.97 \pm 0.30$ & $1.94657 \pm 0.00134$ & $4.399 \pm 0.309$ & $-0.535 \pm 0.013$ \\
$6008.50 \pm 0.36$ & $1.94410 \pm 0.00134$ & $4.266 \pm 0.309$ & $-0.668 \pm 0.021$ \\
$7011.75 \pm 0.38$ & $1.93783 \pm 0.00134$ & $3.805 \pm 0.309$ & $-1.129 \pm 0.013$ \\
$8010.64 \pm 0.46$ & $1.91092 \pm 0.00132$ & $3.352 \pm 0.311$ & $-1.582 \pm 0.010$ \\
$9009.18 \pm 0.31$ & $1.86450 \pm 0.00128$ & $3.061 \pm 0.308$ & $-1.873 \pm 0.029$ \\
$9514.25 \pm 0.54$ & $1.83600 \pm 0.00126$ & $2.881 \pm 0.307$ & $-2.053 \pm 0.035$ \\
\hline
\end{tabular}

Table 11: Effective length results for Endpack 8

\begin{tabular}{|r|r|r|r|}
\hline current, $\mathrm{A}$ & $B_{0} / i$, gauss $/ \mathrm{A}$ & $L_{\text {eff }}, \mathrm{mm}$ & $\Delta L_{\text {eff }}, \mathrm{mm}$ \\
\hline $497.00 \pm 0.38$ & $1.93467 \pm 0.00133$ & $2.670 \pm 0.329$ & $0.313 \pm 0.053$ \\
$1495.21 \pm 0.29$ & $1.94619 \pm 0.00134$ & $2.357 \pm 0.319$ & $0.000 \pm 0.000$ \\
$2998.13 \pm 0.51$ & $1.94878 \pm 0.00134$ & $2.171 \pm 0.317$ & $-0.186 \pm 0.011$ \\
$4001.71 \pm 0.48$ & $1.94743 \pm 0.00134$ & $2.244 \pm 0.313$ & $-0.113 \pm 0.053$ \\
$5005.77 \pm 0.43$ & $1.94722 \pm 0.00134$ & $1.960 \pm 0.315$ & $-0.397 \pm 0.017$ \\
$6008.46 \pm 0.50$ & $1.94498 \pm 0.00134$ & $1.804 \pm 0.315$ & $-0.553 \pm 0.016$ \\
$7011.57 \pm 0.52$ & $1.93865 \pm 0.00133$ & $1.347 \pm 0.316$ & $-1.010 \pm 0.010$ \\
$8010.57 \pm 0.57$ & $1.91225 \pm 0.00131$ & $0.875 \pm 0.318$ & $-1.482 \pm 0.004$ \\
$9009.18 \pm 0.53$ & $1.86608 \pm 0.00128$ & $0.655 \pm 0.313$ & $-1.702 \pm 0.030$ \\
$9513.85 \pm 0.29$ & $1.83772 \pm 0.00126$ & $0.504 \pm 0.312$ & $-1.853 \pm 0.031$ \\
\hline
\end{tabular}

Table 12: Effective length results for Endpack 9 


\begin{tabular}{|r|r|r|r|}
\hline current, $\mathrm{A}$ & $B_{0} / i$, gauss $/ \mathrm{A}$ & $L_{\text {eff }}, \mathrm{mm}$ & $\Delta L_{\text {eff }}, \mathrm{mm}$ \\
\hline $496.89 \pm 0.40$ & $1.93286 \pm 0.00133$ & $2.733 \pm 0.327$ & $0.095 \pm 0.063$ \\
$1495.14 \pm 0.29$ & $1.94337 \pm 0.00134$ & $2.638 \pm 0.323$ & $0.000 \pm 0.000$ \\
$2998.14 \pm 0.17$ & $1.94615 \pm 0.00134$ & $2.397 \pm 0.320$ & $-0.240 \pm 0.012$ \\
$4001.57 \pm 0.28$ & $1.94544 \pm 0.00134$ & $2.352 \pm 0.322$ & $-0.285 \pm 0.034$ \\
$5005.57 \pm 0.37$ & $1.94473 \pm 0.00134$ & $2.177 \pm 0.317$ & $-0.461 \pm 0.061$ \\
$6008.16 \pm 0.37$ & $1.94296 \pm 0.00133$ & $1.940 \pm 0.317$ & $-0.698 \pm 0.030$ \\
$7011.78 \pm 0.26$ & $1.93647 \pm 0.00133$ & $1.501 \pm 0.312$ & $-1.137 \pm 0.088$ \\
$8010.29 \pm 0.30$ & $1.91009 \pm 0.00131$ & $1.078 \pm 0.316$ & $-1.560 \pm 0.058$ \\
$9008.98 \pm 0.36$ & $1.86432 \pm 0.00128$ & $0.827 \pm 0.310$ & $-1.811 \pm 0.054$ \\
$9513.83 \pm 0.51$ & $1.83568 \pm 0.00126$ & $0.759 \pm 0.312$ & $-1.879 \pm 0.054$ \\
\hline
\end{tabular}

Table 13: Effective length results for Endpack 10

\section{Discussion of Results}

These results have converged to an endpack design which meets the requirements for the Main Injector. Let us summarize here the various issues which have been revealed by these studies. Note that the reference for all measurements of $z$ is the outer end of the endpack. Endpack 1 began with a glued stack of laminations which was machined using numerically controlled milling according to the specification provided initially by Stan Snowdon. Endpack 2 was created by modifying the shape of single laminations (nibbling) prior to stacking. This is the technique to be implemented for magnet production and was to be prototyped in this way. It was intended to provide a stepwise approximation to Endpack 1. Endpack 3 was created in the same fashion but with the goal of creating a Borda profile. Endpacks 5-10 were created from Endpack 2 by machining operations. These machining operations were designed, however, to approximate the nibbling which would be done in production.

Looking first at the Borda profile studies using Endpacks 3 and 4, we see that the approximation used for that profile results in a much shorter physical region in $z$ for the transition from body to end. This results in the large positive value of $L_{\text {eff }}$ shown in Figure 4. The large change in $\Delta L_{\text {eff }}$ for Endpack 3 caused us to examine possible sources which might not be easily revealed by the 3-D modeling which was used to predict the 
end shape. To examine possible saturation effects near the coil which would be associated with the added flux which extends into the iron-free region, we constructed a 1.5" thick ring of laminations from which the pole portions had been removed. Installing this in the region from $z=-1.5$ to $z=0$ provided a lower reluctance path for this flux. The improvement of about $30 \%$ in the performance suggests that this effect is not negligible. Since this would be an expensive change in the magnet design, and since it did not result in a satisfactory $\Delta L_{\text {eff }}$, we did not pursue it. Examination of the $L_{\text {eff }}$ results shown in Figure 4 indicates the result that the $\Delta L_{\text {eff }}$ improvement appears to come from smaller lengths at low field rather than less saturation.

The Rogowsky profile was successively approximated by a single cut (Endpacks 1 and 2) and by a cut plus a series of steps (Endpack 5). Endpack 5 was created from the existing Endpack 2 by modifying the gap at $x=0$ in a series of steps in $z$. The resulting profile included laminations for which the iron existed only at distances far from the midplane which reduced $L_{\text {eff }}$ without any significant benefit for the field shape or the effective length variation. Following the successful demonstration of the satisfactory effective length variation of the Rogowsky profile approximation of Endpack 5 , nine of these laminations were removed and additional body laminations added to maintain the steel length. The measurements indicate that about $11 \mathrm{~mm}$ were added to the effective length of the end from adding about 13.5 mm of body laminations. In addition, examination of the profile in the $y-z$ plane reveals that Endpack 6 is a better approximation to the Rogowsky profile. Modifications to create Endpacks 7-10 involved only modifications of the laminations at $x \neq 0$, which were designed to produce improvements in the transverse field behavior. They also improved the $\Delta L_{\text {eff } f}$ behavior.

We can expect small changes when implementing this design in production magnets. The matching of bolted endpack halves to each other and to the body was much less perfect than will be the case in production. In particular, the spring created by the lamination stack must be overcome at the end by a net bowing of the end laminations. This bowing makes it difficult to match to the stacked end of the magnet but the net displacements will have negligible effect of the final shape of the field. Endpacks were mounted with the constraint of minimizing the gap errors on the top-to-bottom parting plane (reduced to less than 0.005") which left larger gaps for some assemblies between the endpack and the body steel. Additional small endpack design changes will be implemented to improve coil clearances. Endpack 11 will be constructed with laminations of this iterated design to confirm these small changes. 


\section{Acknowledgments}

The authors thank Dave Hartness, Shree Agrawal, and Mark Thompson for their excellent work in performing the measurement activities on these endpacks. We also thank Steve Helis for his efforts on construction of the probe. Additional assistance was provided by Butch Bianchi, Jim Garvey, Harold Stahl, and Peter Mazur.

\section{References}

[1] Pertinent details regarding the Main Injector Dipole may be found in the Conceptual Design Report for the Fermilab Main Injector, Rev. 2.3, April 1990.

[2] H. Glass et. al., "Field Shape Measurements of Main Injector Dipole Endpacks," MTF-92-019.

[3] F. Ostiguy, private communication.

[4] F. Ostiguy, private communication.

[5] D. Harding et. al., "Design and Measurements of Prototype Main Injector Dipole Endpacks," (in preparation), to be presente at 1993 Particle Accelerator Conference, Washington, DC.

[6] At present, on-line documentation describing the FLATCOIL data acquisition program can be found in the MSR\$ROOT:[DOC] directory on node MDTF00:: 

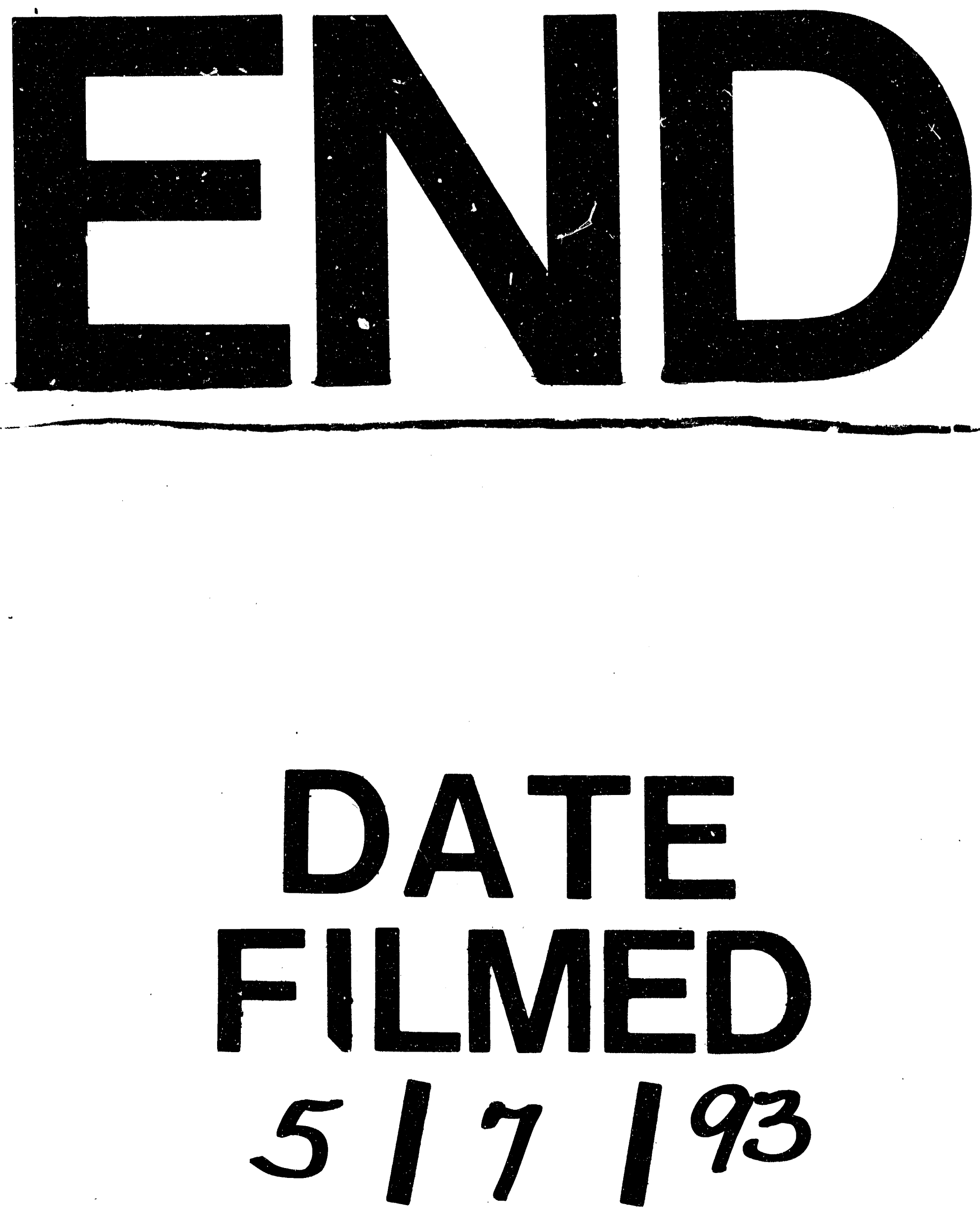
